\title{
COMPLEX CONSTRAINED CRB AND ITS APPLICATION TO SEMI-BLIND MIMO AND OFDM CHANNEL ESTIMATION
}

\author{
Aditya K. Jagannatham and Bhaskar D. Rao \\ University of California, SanDiego \\ 9500 Gilman Drive, La Jolla, CA 92093-0407 \\ e-mail: \{ajaganna,brao\}@ucsd.edu
}

\begin{abstract}
We derive an expression for the Cramer-Rao lower bound (CRB) on the covariance of unbiased estimators of a constrained complex parameter vector and demonstrate key applications of the theory. In the first part, motivated by the result in [1] we introduce an important extension to the set of constraints on the complex parameters. This extension then leads to a simple expression for the complex constrained CRB similar to the result in [2]. This CRB framework is employed in the context of estimation of a wireless channel matrix $H$. We demonstrate that the channcl estimation error lower bound is directly proportional to the number of unconstrained parameters in $H$. The usefulness of these results is illustrated by their application to two different estimation problems arising in wireless communications. The first is whitening-rotation based semi-blind MIMO channel cstimation and the second pertains to time versus frequency domain channel estimation in OFDM systems.
\end{abstract}

\section{INTRODUCTION}

The CRB serves as an important tool in the performance evaluation of estimators which arise frequently in the fields of communications and signal processing. Most problems involving the CRB are formulated in terms of unconstrained real parameters [3]. Two useful developments of the CRB theory have been presented in later rescarch. The first being a CRB formulation for unconstrained complex parameters given in [1]. A second result is the development of the CRB theory for constrained real parameters [2]. However, in applications such as semi-blind channel estimation one is faced with the estimation of constrained complex parameters. Using the calculus of complex derivatives as is often done in signal processing applications, considerable insight and simplicity can be achieved by working with the complex vector parameter as a single entity $[3,4]$. We thus present an extension of the result in [2] for the case of constrained complex parameters. Its application is illustrated

This work was supported by CoRe research grant Cor00-10074 in the context of semi-blind channel estimation and also to OFDM based communication systems.

\section{CRB FOR COMPLEX PARAMETERS WITH CONSTRAINTS}

Consider the complex parameter vector $\bar{\gamma} \in \mathbb{C}^{n \times 1}$. Let $\bar{\gamma} \triangleq$ $\bar{\alpha}+j \bar{\beta}$ such that the real and imaginary parameter vectors $\bar{\alpha}, \bar{\beta} \in \mathbb{R}^{n \times 1}$ and $\bar{\xi} \triangleq\left[\bar{\alpha}^{T}, \bar{\beta}^{T}\right]^{T}$. Assume that the likelihood function of the (possibly complex) observation vector $\bar{\omega} \in \Omega$ parameterized by $\bar{\xi}$ is $s(\bar{\omega} ; \bar{\xi})$. Let $\hat{\bar{\xi}}: \Omega \rightarrow \mathbb{R}^{2 n \times 1}$ be given as $\hat{\bar{\xi}} \triangleq\left[\hat{\bar{\alpha}}^{T}, \hat{\beta}^{T}\right]^{T}$, where $\hat{\bar{\alpha}}, \hat{\bar{\beta}}$ are unbiased estimators of $\bar{\alpha}, \bar{\beta}$ respectively. In the foregoing analysis, we define the gradient $\frac{d r(\bar{\alpha})}{d \bar{\alpha}} \in \mathbb{R}^{1 \times n}$ of a scalar function $r(\bar{\alpha})$ as a row vector: $\frac{d r(\bar{\alpha})}{d \bar{\alpha}} \triangleq\left[\frac{d r(\bar{\alpha})}{d \alpha_{1}}, \frac{d r(\bar{\alpha})}{d c k_{2}}, \ldots, \frac{d r(\bar{\alpha})}{d \alpha_{n}}\right]$.

Let $\bar{\theta} \in \mathbb{C}^{2 \pi \times]}$ be defined as in [1] by $\bar{\theta} \triangleq\left[\bar{\gamma}^{T}, \bar{\gamma}^{H}\right]^{T}$. Suppose now that the $l$ complex constraints on $\bar{\theta}$ are given as

$$
\mathbf{h}(\bar{\theta})=\mathbf{0},
$$

i.e. $\mathbf{h}(\bar{\theta}) \in \mathbb{C}^{l \times 1}$. We then construct an extended constraint set (of possibly redundant constraints) $f(\bar{\theta}) \in \mathbb{C}^{2 l \times 1}$ as

$$
\mathbf{f}(\bar{\theta}) \triangleq\left[\begin{array}{c}
\mathbf{h}(\bar{\theta}) \\
\mathbf{h}^{*}(\bar{\theta})
\end{array}\right]=\mathbf{0}
$$

An important observation from (2) above is that symmetric complex constraints on these parameters are treated as disjoint. For instance, given the orthogonality of complex parameter vectors $\bar{\eta}_{1}, \bar{\eta}_{2}$, i.e. $\bar{\eta}_{1}^{H} \bar{\eta}_{2}=0$, the symmetric constraint $\bar{\eta}_{2}^{H} \bar{\eta}_{1}=0$ is to be treated as an additional complex constraint and hence $\mathrm{f}(\bar{\theta})=\left[\bar{\eta}_{1}^{H} \bar{\eta}_{2}, \bar{\eta}_{2}^{H} \bar{\eta}_{1}\right]^{T}$. The extension of the constraints is akin to the extension of the parameter set from $\bar{\gamma}$ to $\bar{\theta}=\left[\bar{\gamma}^{T}, \bar{\gamma}^{H}\right]^{T}$ called for when dealing with complex parameters. Let $F(\bar{\theta}) \triangleq \frac{\partial \mathrm{r}\{\bar{\theta})}{\partial \theta}$ and $\operatorname{rank}(F(\bar{\theta}))=k<2 n$. Hence there exists a $U \in \mathbb{C}^{2 n \times 2 n-k}$ such that $U$ forms an orthonormal basis for the nullspace of 
$F(\bar{\theta})$ i.e. $F(\bar{\theta}) U=0$. Let $p(\bar{\omega} ; \bar{\theta})$ be the reparameterized likelihood $s(\bar{\omega} ; \bar{\alpha}, \bar{\beta})$ of the observed data and define $\Delta$ as

$$
\Delta \triangleq \frac{\partial \ln p(\bar{\omega} ; \bar{\theta})}{\partial \bar{\theta}}=\left[\frac{\partial \ln s(\bar{\omega} ; \bar{\xi})}{\partial \bar{\gamma}}, \frac{\partial \ln s(\bar{\omega} ; \bar{\xi})}{\partial \bar{\gamma}^{*}}\right]^{T},
$$

where the last equation follows from the definition of $p(\bar{\omega} ; \bar{\theta})$. Let $J=\mathrm{E}\left\{\Delta^{*} \Delta^{T}\right\}$ denote the Fisher information matrix (FIM) for the unconstrained estimation of $\bar{\theta}$. Also assume that

\section{A.1: The real parameter vector $\bar{\xi}$ satisfies the desired regu- larity conditions as in [2]}

We now state the result for the CRB of the constrained complex estimator $\hat{\bar{\theta}}$.

Theorem 1. Under assumption A.I and constraints given by (1), the CRB for estimation of the constrained parameter $\tilde{\theta} \in \mathbb{C}^{2 n \times 1}$ is then given as

$$
\mathrm{E}\left\{(\hat{\bar{\theta}}-\bar{\theta})(\hat{\hat{\theta}}-\bar{\theta})^{H}\right\} \geq U\left(U^{H} J U\right)^{-1} U^{I H} .
$$

Proof: A proof of the above result can be found in [5].

\section{SEMI-BLIND MIMO CHANNEL ESTIMATION}

\subsection{Problem Formulation}

We consider in this section the problem of pilot assisted semi-blind estimation of a complex MIMO channel matrix $H \in \mathbb{C}^{r \times t}$ (\# receive antennas $=r$,\# transmit antennas $=t$ ). Let a total of $L$ pilot symbols be transmitted. The channel input-output relation is represented as

$$
\mathbf{y}_{k}=H \mathbf{x}_{k}+\mathbf{v}_{k} \quad, \quad k=1,2, \ldots, L,
$$

where $\mathrm{y}_{k} \in \mathbb{C}^{r \times 1}, \mathrm{x}_{k} \in \mathbb{C}^{t \times 1}$ are the received and transmitted signal vectors at the $k$-th time instant. $\mathbf{v}_{k} \in \mathbb{C}^{r \times 1}$ is spatio-temporally uncorrelated Gaussian noise such that $\mathrm{E}\left\{\mathbf{v}_{k} \mathbf{v}_{k}^{H}\right\}=o_{n}^{2} \mathbf{I}$. The received symbol vectors can be stacked as $\tilde{Y}_{p} \triangleq\left[\tilde{\mathbf{y}}_{1}, \tilde{\mathbf{y}}_{2}, \ldots, \tilde{\mathbf{y}}_{L}\right]$. Let $X_{p}$ be defined anal. ogously by stacking the transmitted symbol vectors. The next result then relates the estimation accuracy of $H$ to the number of un-constrained parameters in it.

Theorem 2. Under conditions described above, if the pilot sequence $X_{p}$ is orthogonal i.e. $X_{p} X_{p}^{H}=\sigma_{s}^{2} L \mathrm{I}$, the minimum estimation error in $H$ is directly proportional to $\Lambda$ the number of un-constrained parameters required to describe $H$ and in fact,

$$
\mathrm{E}\left\{\|\hat{H}-H\|_{F}^{2}\right\} \geq \frac{\sigma_{n}^{2}}{2 \sigma_{s}^{2} L} \Lambda
$$

Proof: A proof of the above result can be found in [6].

A general complex channel matrix $H$ has $\Lambda_{H}=2 r t$ parameters. However, $H$ can be factorized using its singular value decomposition (SVD) as $H=P \Sigma Q^{H}$ where $P \in$ $\mathbb{C}^{r \times t}, Q \in \mathbb{C}^{t \times t}$ is unitary such that $Q^{H} Q=Q Q^{H}=\mathbf{I}$, $\Sigma=\operatorname{diag}\left(\sigma_{1}, \sigma_{2}, \ldots, \sigma_{i}\right), \sigma_{i}>0 . P, \Sigma$ can be estimated using blind techniques. We then employ the pilot data exclusively to estimate the constrained orthogonal matrix $Q$ which has $\Lambda_{Q}=t^{2}$ parameters. The semi-blind estimate of $H$ is then given as $\hat{H}=P \Sigma \hat{Q}^{H}$. More about the significance of such an estimation scheme can be found in [7]. Hence by an application of theorem(2), the ratio of the estimation error bounds of the semi-blind technique which estimates a constrained matrix $Q$, to a training based technique that estimates the un-constrained $H$ matrix is given as

$$
\mathcal{G}=10 \log _{10}\left(\frac{\Lambda_{H}}{\Lambda_{Q}}\right) \mathrm{dB}=10 \log _{10}\left(\frac{2 r}{t}\right) \mathrm{dB} .
$$

The above expression holds with equality when the CRB is achievable. Thus, for a size $8 \times 4$ channel matrix, $\mathcal{G}=$ $6 \mathrm{~dB}$ which implies a significant improvement in estimation accuracy.

\subsection{Cramer-Rao Bound}

Let $\tilde{\mathbf{y}}_{k}=P^{H} \mathbf{y}_{k}, \tilde{\mathbf{v}}_{k}=P^{H} \mathbf{v}_{k}$. Denote by $\mathbf{q}_{i}$ the $\mathrm{i}$-th column of the matrix $Q$. The unconstrained input-output relation for each $\mathbf{q}_{i}$ can be written as

$$
\tilde{\mathbf{y}}_{k, i}=\sigma_{i} \mathbf{x}_{i}^{H} \mathbf{q}_{i}+\tilde{\mathbf{v}}_{k, i},
$$

where $\mathbf{y}_{k, i}$ denotes the $\mathrm{i}$-th element of $\mathrm{y}_{k}$ and analogously for $\mathbf{v}_{k, i}$. Define the desired parameter vector to be estimated $\bar{\theta} \triangleq\left[\operatorname{vec}(Q), \operatorname{vec}\left(Q^{*}\right)\right]^{T}$. It is shown in [5] that for the above problem the matrices $F(\bar{\theta}) \in \mathbb{C}^{t^{2} \times 2 t^{2}}, U \in$ $\mathbb{C}^{2 t^{2} \times t^{2}}$ can be written explicitly and are given as

$$
\begin{gathered}
F(\bar{\theta})=\left[\begin{array}{llllllll}
\mathbf{q}_{1}^{H} & 0 & 0 & \ldots & \mathbf{q}_{1}^{T} & 0 & 0 & \ldots \\
0 & \mathbf{q}_{1}^{H} & 0 & \ldots & \mathbf{q}_{2}^{T} & 0 & 0 & \ldots \\
\mathbf{q}_{2}^{H} & 0 & 0 & \ldots & 0 & \mathbf{q}_{1}^{T} & 0 & \ldots \\
0 & \mathbf{q}_{2}^{H I} & 0 & \ldots & 0 & \mathbf{q}_{2}^{T} & 0 & \ldots \\
\mathbf{q}_{3}^{H} & 0 & 0 & \ldots & 0 & 0 & \mathbf{q}_{1}^{T} & \ldots \\
0 & 0 & \mathbf{q}_{1}^{H} & \ldots & \mathbf{q}_{3}^{T} & 0 & 0 & \ldots \\
\vdots & \vdots & \vdots & \ddots & \vdots & \vdots & \vdots & \ddots
\end{array}\right] \\
U=\frac{1}{\sqrt{2}}\left[\begin{array}{cccccc}
\mathbf{q}_{1} & 0 & \mathbf{q}_{2} & 0 & \mathbf{q}_{3} & \ldots \\
0 & \mathbf{q}_{1} & 0 & \mathbf{q}_{2} & 0 & \ldots \\
0 & 0 & 0 & 0 & 0 & \ldots \\
\vdots & \vdots & \vdots & \vdots & \vdots & \ddots \\
-\mathbf{q}_{1}^{*} & -\mathbf{q}_{2}^{*} & 0 & 0 & 0 & \ldots \\
0 & 0 & -\mathbf{q}_{1}^{*} & \mathbf{q}_{2}^{*} & 0 & \ldots \\
0 & 0 & 0 & 0 & -\mathbf{q}_{1}^{*} & \ldots \\
\vdots & \vdots & \vdots & \vdots & \vdots & \ddots
\end{array}\right] .
\end{gathered}
$$


The simplistic and insightful nature of the above matrices in terms of the orthogonal parameter vectors is particularly appealing and illustrates the efficacy of using the complex CRB. From $\mathrm{Eq}(8)$ and using the results for least-squares estimation [3] the Fisher information matrix $J(\bar{\theta}) \in \mathbb{C}^{2 t^{2} \times 2 t^{2}}$ for the unconstrained case is given by the block diagonal matrix

$$
J(\bar{\theta})=\frac{1}{\sigma_{\pi}^{2}} \mathbf{I}_{2 \times 2} \otimes \Sigma^{2} \otimes X_{p} X_{p}^{H} .
$$

The complex constrained CRB for the parameter vector $\vec{\theta}$ is then obtained by substituting these matrices in (4). For an orthonormal pilot sequence $X_{p}$ (i.e. $X_{p} X_{p}^{H}=L \sigma_{s}^{2} \mathrm{I}$ ) the CRB matrix for $\hat{Q}$ (and hence that of $\hat{H}$ ) can be explicitly written and the lower bound for the variance in the estimate of the $k, l$-th element of $H$ is shown in [6] to be given as

$$
\mathrm{E}\left\{\left|\hat{H}_{k l}-H_{k l}^{2}\right|\right\} \geq \frac{\sigma_{n}^{2}}{\sigma_{s}^{2} L} \sum_{i=1}^{t} \sum_{j=1}^{t} \frac{\sigma_{i}^{2}\left|P_{k i} Q_{l j}\right|^{2}}{\sigma_{j}^{2}+\sigma_{i}^{2}}
$$

where $P_{k i}, Q_{l j}$ are the $k, i$-th and $l, j$-th elements of $P, Q$ respectively.

\subsection{Estimation of $Q$}

$\hat{Q}$, the ML estimate of $Q$, is given as a solution to the cost function

$$
\hat{Q}=\arg \min _{Q}\left\|\tilde{Y}_{p}^{H}-X_{p}^{H} Q \Sigma\right\|^{2} \text { subject to } Q Q^{H}=\mathbf{I}
$$

where the norm $\|-\|$ is the matrix Frobenius norm. From $[7$, 8,9], the constrained estimate $\hat{Q}$ employing an orthonormal pilot sequence $X_{p}$, i.e. $X_{p} X_{p}^{I I}=\sigma_{s}^{2} L \mathbf{I}$, is given as

$$
\hat{Q}=P_{p} R_{p}^{H} \text { where } P_{p} \Sigma_{p} R_{p}^{H}=\operatorname{svd}\left(X_{p} \tilde{Y}_{p}^{H} \Sigma\right),
$$

and $\hat{H}_{s b}$ the semi-blind estimate of $H$ is given as

$$
\hat{H}_{s b}=P \Sigma \hat{Q}^{H}
$$

Denoting by $X_{p}^{\dagger}$ the Moore-Penrose pseudo-inverse of $X_{p}$, the exclusive training based ML estimate of $H$ is given as

$$
\hat{H}_{t}=Y_{p} X_{p}^{\dagger}
$$

\subsection{Simulation Results}

Our simulation set-up consists of an $8 \times 4$ MIMO channel $H$. The source symbol vectors $\mathrm{x} \in \mathbb{C}^{4 \times 1}$ are assumed to be drawn from a BPSK constellation and the orthonormality condition is achieved by using the Hadamard structure. The transmitted pilot was assumed to be of length $L=12 \mathrm{sym}$ bols. Fig. 1. shows the total MSE in estimation of $H$ using the semi-blind estimate in (13) and the exclusive training

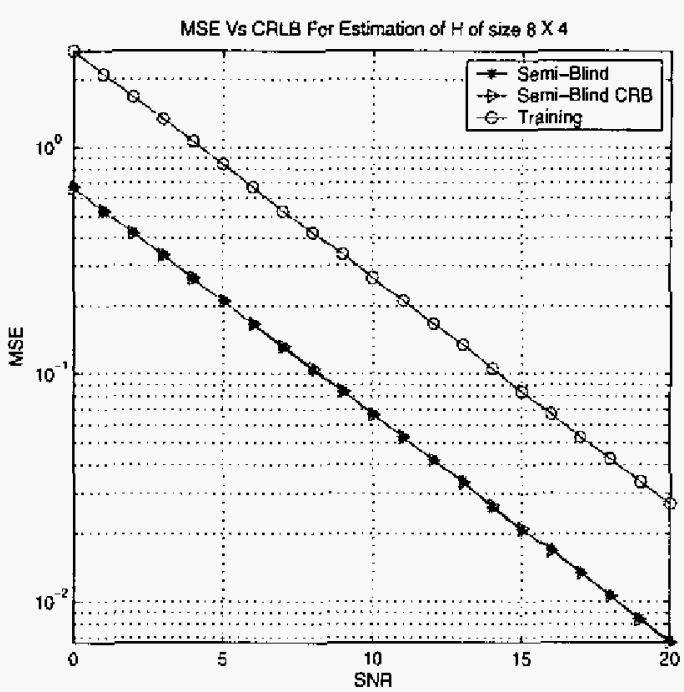

Fig. 1. Computed MSE Vs SNR. $\|\hat{H}-H\|_{F}^{2}$

based least squares estimate given in (14). Also shown is the CRB of the semi-blind estimate computed from (10). The semi-blind ML estimate $\hat{H}_{s b}$ can be seen to achieve a performance close to the $\mathrm{CRB}$ and it is also seen to be $(\mathcal{G}=) 6$ dB more efficient than the exclusive training based estimate $\hat{H}_{t}$, as can be inferred from (7).

\section{OFDM CHANNEL ESTIMATION}

We demonstrate another application of constrained estimation by considering a problem that arises in the context of channel estimation in Orthogonal frequency division multiplexing (OFDM) communication systems. The problem of time vs frequency domain channel estimation for an OFDM based communication system has been detailed in $[10,11]$. It has been shown there in that when the number of subcarriers $K$ exceeds the numbers of taps $L$ in the channel impulse response (CIR), the time domain least squares channel estimate (TLSE) is more accurate than the frequency domain least squares estimate (FLSE). Indeed, we demonstrate below that this result follows as an immediate consequence of the complex constrained CRB theory developed above.

\subsection{Problem Description}

Employing notation in [10], let the complex baseband channel from the transmitter to the receiver be modelled by a tapped delay line as

$$
h(\tau, t)=\sum_{l=0}^{L-1} h_{l}(t) \delta\left(\tau-l T_{s}\right)
$$


where $L$ is the number of taps in the channel and is known. Let $K$ denote the number of subcarriers and $\mathrm{p} \triangleq\left[a_{0}, a_{1}, \ldots\right.$, $\left.a_{K-1}\right]^{T}$ be the pilot signal known at the receiver. Denoting the discrete time CIR as $\mathbf{h}=\left[h_{0}, h_{1}, \ldots, h_{L-1}\right]^{T}$, the cyclic-prefix extended OFDM communication system can be modeled as

$$
\mathbf{r}=\mathbf{a h}+\mathbf{n}
$$

where $\mathbf{r}, \mathbf{n} \in \mathbb{C}^{K \times 1}$ are the received symbol vector and additive whitc Gaussian noise respectively. The matrix $a \in$ $C^{K \times L}$ is constructed from the pilot symbols as

$$
a \triangleq\left[\begin{array}{ccccc}
a_{0} & a_{K-1} & a_{K-2} & \ldots & a_{K-L+1} \\
a_{1} & a_{0} & a_{K-1} & \ldots & a_{K-L+2} \\
\vdots & \vdots & \vdots & \ddots & \vdots \\
a_{L-1} & a_{L-2} & a_{L-3} & \ldots & a_{0} \\
\vdots & \vdots & \vdots & \ddots & \vdots \\
a_{K-1} & a_{K-2} & a_{K-3} & \ldots & a_{K-L}
\end{array}\right]
$$

$\hat{h}$, the LS estimate of $h$ is given as

$$
\hat{h}=\left(a^{H} a\right)^{-1} a^{H} r .
$$

The frequency domain equivalent of the system in (16) can be obtained by computing the DFT of both sides as

$$
\mathbf{R}=F \mathbf{r}=F \mathbf{a h}+F \mathbf{n},
$$

where $F \in \mathbb{C}^{K \times K}$ is given as

$$
F=\left[\begin{array}{ccc}
W^{00} & \ldots & W^{0(K-1)} \\
\vdots & \ddots & \vdots \\
W^{(K-1) 0} & \ldots & W^{(K-1)(K-1)}
\end{array}\right]
$$

and $W^{i l} \triangleq e^{-j \frac{2 \pi i l}{\kappa}}$. The system in (19) is then given as

$$
\mathbf{R}=A \mathbf{H}+\mathbf{N} \text {, }
$$

where $A \stackrel{A}{=} \operatorname{diag}(F p) \in \mathbb{C}^{K \times K}, \mathbf{N} \triangleq F \mathrm{n}$ and $\mathbf{H} \triangleq \tilde{F} \mathrm{~h}$ where $\tilde{F}$ is the left $K \times L$ sub-matrix of $F$. The unconstrained least squares estimate $\hat{H}$, which is also the FLSE, is therefore given as

$$
\hat{H}_{f}=\left(A^{H} A\right)^{-1} A^{H} R \text {. }
$$

However, the parameter vector $\mathbf{H}$ is a constrained parameter vector and in fact, the constraints on $\mathbf{H}$ are given as

$$
\mathbf{f}(\mathbf{H}) \triangleq \overline{\bar{F}}^{H} \mathbf{H}=\mathbf{0}
$$

where $\overline{\tilde{F}}$ is the right $K \times(K-L)$ sub-matrix of $F$. Therefore, from (23), it can be seen that the number of constraints on $H$ is $K-L$. Hence, even though $\mathbf{H}$ contains $K$ complex parameters ( $2 K$ real parameters), it only contains $L$
$(<K)$ un-constrained complex parameters $(2 L$ real parameters) and these un-constrained parameters are in fact the elements of the parameter vector $h . \mathbf{H}$ is given as a function of its un-constrained parameters as $\mathbf{H}=\tilde{F}$ h. Thus, an alternative constrained technique to estimate $\mathbf{H}$, based on the estimate in (18) is given as,

$$
\hat{H}_{t}=\tilde{F} \hat{h}
$$

which is also the TLSE of $\mathbf{H}$. Assuming a constant power spectrum as in [10], $A^{F I} A=\mathbf{I}$. Hence, the pilot orthogonality requirement of theorem 2 is satisfied and in fact, the $\mathrm{CRB}$ is exactly achievable since the estimation problem in this case involves a linear least squares cost function and the noise is Gaussian [3]. Therefore, from theorem 2, the ratio of the estimation error of the FLSE in (22) to the estimation error in the TLSE in (24) is precisely given by the ratio of the number of parameters to the number of un-constrained parameters as

$$
\frac{\mathrm{E}\left\{\left\|\hat{H}_{f}-H\right\|_{F}^{2}\right\}}{\mathrm{E}\left\{\left\|\hat{H}_{t}-H\right\|_{F}^{2}\right\}}=\frac{K}{L}
$$

as reported in [10], where the above conclusion was reached after an explicit computation of the covariance matrices of the time domain and frequency domain estimation schemes. Thus, the constrained parameter framework and particularly theorem 2 provides a powerful framework, where results such as the one in (25) can be deduced by just reckoning the nember of un-constrained parameters, thus avoiding explicit computation of the error covariance matrices.

\subsection{Simulation results}

Our simulation setup consisted of an OFDM system with $K=40$ subcarriers and $L=5$ taps. The channel $h$ was generated as a complex Gaussian vector of zero mean independent entries and with the variance of real and imaginary parts equal to 0.5 . The time domain and frequency domain channel estimates were found as given in (24) and (22) respectively. The experiment was repeated for 1000 iterations at different SNRs in the range $2-14 \mathrm{~dB}$. The mean estimation error vs SNR is given in Fig.2. It can be seen the the time domain estimate is more accurate than the frequency domain estimate. Also, the ratio of the estimation error of the FLSE to TLSE is precisely $10 \log _{10}\left(\frac{K}{L}\right)=6 \mathrm{~dB}$.

\section{CONCLUSIONS}

An expression for the Cramer-Rao lower bound on the covariance of unbiased estimators of a constrained complex 


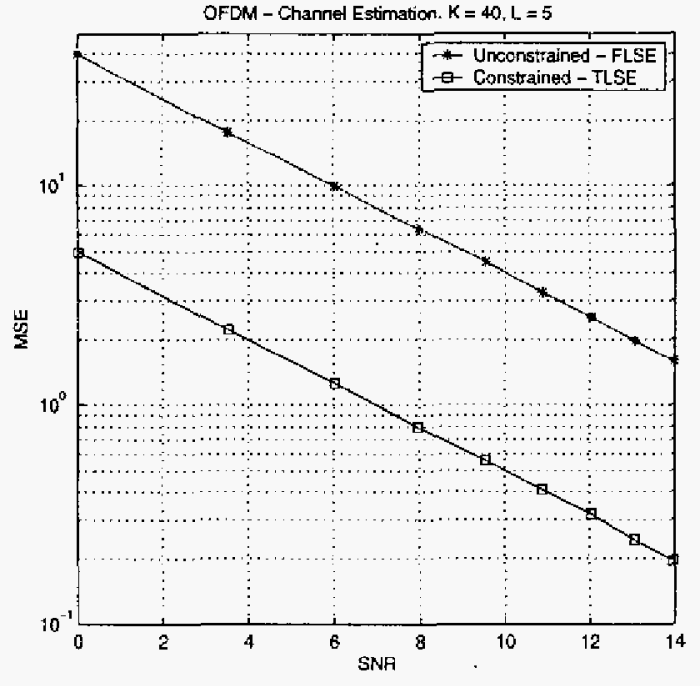

Fig. 2. Constrained $V_{s}$. unconstrained channel estimation for OFDM.

parameter vector has been presented. Employing this result, we have demonstrated that the channel estimation error lower bound is directly proportional to the number of unconstrained parameters in $H$, the channel matrix. The applicability of this framework is shown in the context of two different wireless channel estimation problems. The first scenario is whitening-rotation based semi-blind MIMO channel estimation and the second pertains to time versus frequency domain channel estimation in OFDM systems.

\section{REFERENCES}

[1] A. van den Bos, "A cramer-rao lower bound for complex parameters," IEEE Transactions on Signal Processing, vol. 42, no. 10, pp. 2859, october 1994.

[2] P. Stoica and B. C. Ng, "On the cramer-rao bound under parametric constraints," IEEE Signal Processing Letters, vol. 5, no. 7, pp. 177-[79, Ju] 1998.

[3] S. M. Kay, Fundamentals of Statistical Signal Processing,Vol I: Estimation Theory, Prentice Hall PTR, first edition, 1993.

[4] D. H. Brandwood, "A complex gradient operator and its application in adaptive array theory," IEE Proc., vol. 130, no. 1, pp. 11-16, Feb. 1983.

[5] A. K. Jagannatham and B. D. Rao, "Cramer-rao lower bound for constrained complex parameters," Accepted for publication in IEEE Signal Processing Letters.
[6] A. K. Jagannatham and B. D. Rao, "Whitening rotation based semi-blind MIMO channel estimation," Submitted to IEEE Transactions on Signal Processing.

[7] A. K. Jagannatham and B. D. Rao, "A semi-blind technique for MIMO channel matrix estimation," in Proc. of IEEE Workshop on Signal Processing Advances in Wireless Communications (SPAWC 2003), \# 582, Rome, Italy, 2003.

[8] G. H. Golub and C. F. Van Loan, Matrix Computations, Johns Hopkins Univ Pr, second edition, 1984.

[9] A. Medles, D. T. M. Slock, and E. De Carvalho, "Lirtear prediction based semi-blind estimation of mimo fir channels," Third IEEE SPAWC, Taoyuan, Taiwan, pp. $58-61$.

[10] Z. Cheng and D. Dahlhaus, "Time versus frequency domain channel estimation for ofdm systems with antenna arrays," Proc. of 6 th International Conference on Signal Processing (ICSP'O2), Beijing. China, vol. 2, pp. 1340-1343, Aug 2002.

[11] J. Heiskala and J. Terry, OFDM Wireless LANs: A Theoretical and Practical Guide, SAMS Publishing, 2002. 